\title{
Las relaciones interpersonales en la transición de los estudiantes de la primaria a la secundaria
}

\section{Interpersonal Relationships in the Transition of Students from Primary to Secondary* Schools}

\author{
Marianella Castro Pérez ${ }^{l}$ \\ Instituto de Estudios Interdisciplinarios de la Niñez y la Adolescencia (CIDE) \\ Universidad Nacional \\ Heredia, Costa Rica \\ nellacp@yahoo.com \\ Mirta Díaz Forbice ${ }^{2}$ \\ División de Educación Rural (CIDE) \\ Universidad Nacional \\ Heredia, Costa Rica \\ mirtadia@gmail.com \\ Hilda Fonseca Solórzano ${ }^{3}$ \\ División de Educología (CIDE) \\ Universidad Nacional \\ Heredia, Costa Rica \\ hildafon@gmail.com \\ Ana Teresa León Sáenz ${ }^{4}$ \\ Instituto de Estudios Interdisciplinarios de la Niñez y la Adolescencia (CIDE) \\ Universidad Nacional \\ Heredia, Costa Rica \\ analeon@racsa.co.cr \\ Lillian Susana Ruíz Guevara ${ }^{5}$ \\ División de Educación Básica (CIDE) \\ Universidad Nacional \\ Heredia, Costa Rica \\ susanaruiz@racsa.co.cr \\ Willy Umaña Fernández ${ }^{6}$ \\ División de Educación para el Trabajo (CIDE) \\ Universidad Nacional \\ Heredia, Costa Rica \\ wumanaf@costarricense.cr
}

Recibido 14 de junio de $2010 \bullet$ Aceptado 31 de agosto de 2010

\footnotetext{
N. of the T.: In Costa Rican education system, primary school comprises from the 1st to 6th grades of the Basic General Education, and secondary school comprises from the 7th to 11th grades.

1 Licenciada en Educación Preescolar y Máster en Administración Educativa, Académica del INEINA y de la División de Educación Básica, Universidad Nacional de Costa Rica.

2 Máster en Educación, Académica de la División de Educación Rural, Universidad Nacional de Costa Rica.

Máster en Educación, Académica de la División de Educología, Universidad Nacional de Costa Rica.

$4 \quad$ Licenciada en Psicología y Máster en Desarrollo del Niño, Directora del INEINA, Universidad Nacional de Costa Rica.

5 Licenciada en Educación Preescolar, Máster en Administración Educativa y Doctora en Educación, Académica de la División de Educación Básica, Universidad Nacional Costa Rica.

6 Licenciado en Planificación Social, académico pensionado de la División de Educación para el Trabajo, Universidad Nacional, Costa Rica.
} 
Resumen. El tema de las relaciones interpersonales que establece el estudiante con sus pares, profesores y padres de familia, durante el periodo de transición de la primaria a la secundaria, es el punto sobre el cual se discute en este artículo, ya que se presentan los resultados más sobresalientes de la investigación titulada Factores de éxito en la transición de sexto grado a sétimo de secundaria, realizada entre los años 2006-2007, en el marco del Programa de investigación: Perfiles, dinámicas y desafíos de la educación costarricense. Esta investigación plantea, como uno de sus objetivos, recabar la opinión del alumnado de $6^{\circ}, 7^{\circ}$ y $8^{\circ}$ años, respecto a lo que consideran como los factores claves para lograr una transición exitosa entre primaria y secundaria. Es un estudio descriptivo y exploratorio, ya que sus resultados permitirán lograr un acercamiento o aproximación al tema y servirá, además, de punto de partida para otros trabajos de investigación vinculados a esta temática. Utiliza técnicas cuantitativas y cualitativas, sin embargo, no es representativa de la situación a nivel nacional. Los hallazgos más importantes permiten concluir que, al ingresar a la secundaria, los estudiantes manifiestan tener buenas relaciones interpersonales con sus padres, sus profesores y sus compañeros, razón por la cual la aprobación y el apoyo que cada uno de ellos les brinde es fundamental para lograr consolidar su posición en el nuevo grupo académico y, por supuesto, para mejorar la percepción que ellos tienen de sí mismos.

Palabras claves. Transición, factores de éxito, relaciones interpersonales.

Abstract. Interpersonal relationships between students and their parents, teachers and peers during the transition from primary* to secondary school is the topic of this paper. It includes the main results of the survey "Factores de éxito en la transición de sexto grado a sétimo de secundaria" (Success factors on the student's transition from $6^{\text {th }}$ to $7^{\text {th }}$ grade), held from 2006 to 2007, in the framework of the research program "Perfiles, Dinámicas y Desafíos de la Educación Formal Costarricense" (Challenges of the Costa Rican formal education). One of the objectives in this research project was to obtain the opinion of students from $6^{\text {th }}, 7^{\text {th }}$ and $8^{\text {th }}$ grades on what they consider to be key factors for a successful transition between primary and secondary education. The results of this descriptive and exploratory study will contribute to a better understanding of the topic, and will also serve as a starting point for further research in this area. Although quantitative and qualitative techniques are used, the study is non-representative of the national situation. The most important findings reveal that upon entering the secondary school, students have good relationships with their parents, teachers and peers, and their approval and support its fundamental for students to consolidate a position in the new academic group and, of course, to improve their self-perception.

Keywords. Transition, success factors, interpersonal relationships.

\section{Introducción}

La investigación titulada Factores de éxito en la transición de sexto a grado a sétimo de secundaria, de la cual se desprenden los datos que se discuten en este artículo, tiene como uno de sus objetivos recabar la opinión del alumnado de $6^{\circ}, 7^{\circ}$ y $8^{\circ}$ años, respecto a lo que ellos y ellas consideran como los factores claves para lograr una transición exitosa entre primaria y secundaria

En el proceso de la transición de la primaria a la secundaria, la bibliografía establece que la ruptura en las relaciones sociales del estudiante es una de las consecuencias más importantes para los alumnos, especialmente porque su círculo más cercano de compañeros se dispersa y la cantidad de docentes aumenta. Con ello se incrementa la necesidad de desarrollar nuevas alianzas y estrategias para relacionarse con cada uno de sus profesores.

Este artículo presenta datos acerca de relaciones interpersonales que establece el estudiante con sus padres, profesores y compañeros, como uno de los factores de éxito en el proceso de transición. Además, devela información del autoconcepto, los valores y sentimientos de un grupo de jóvenes que cursan el sétimo y octavo de secundaria. 
Las temáticas de relaciones interpersonales, autoconcepto y valores se interrelacionan con la intención de profundizar en la importancia del componente afectivo en los procesos de enseñanza y aprendizaje, principalmente durante la transición a la secundaria, momento en el cual los estudiantes sufren declinación en la percepción de sí mismos, lo que repercute en el rendimiento académico.

Por otra parte, este artículo pone en evidencia que el padre de familia cumple una función muy importante para los muchachos que recién inician su vida en la secundaria. Este aspecto exige, por tanto, revalorar el papel de los padres de familia en los procesos de enseñanza y aprendizaje que viven sus hijos y, de manera especial, en el proceso de transición de la primaria a la secundaria.

\section{Referente teórico}

\section{Relaciones entre pares durante el proceso de transición}

La etapa de la adolescencia que enfrentan los estudiantes en su paso a la secundaria se caracteriza por ser una etapa intermedia de la vida, en la cual ellos no son niños, ni tampoco adultos, por tanto, su mundo se vuelve más complejo. De ahí que:

La institución escolar, es a la vez, un refugio para construir un mundo propio al margen de los adultos familiares, al tiempo que representa la continuidad del control por parte de los adultos, así como de los valores y algunas normas de estos. Es un cobijo no tanto por lo que es en sí misma, como por ser un espacio y tiempo para entablar unas relaciones independientes del medio familiar, en que se desarrollan la mayoría de las veces tanto al margen de la familia como del centro escolar. (Gimeno, 1997, p. 87)

Esta nueva cultura juvenil, en la secundaria, dice Gimeno (1997), hace que los alumnos armen redes de amistad y camaradería, en las cuales los valores de la cultura escolar y los provenientes de la familia se ven enfrentados y, en muchas ocasiones, cuestionados. Lo anterior, debido a que el nuevo grupo de amigos y compañeros se convierte en el signo de seguridad, por tanto, se asumen los valores que este grupo de pares ha reelaborado y se hacen explícitos en las relaciones de amistad.

Unido a esto, las relaciones interpersonales que establecen los estudiantes con el sexo opuesto cobran un sentido diferente; además, encuentran mayores espacios para expresarse con amplia libertad, arriesgar iniciativas y relacionarse de igual a igual, sin que existan problemas de mando o superioridad: "En el grupo de iguales el adolescente encuentra lealtad, un clima en el que expresar con confianza conflictos y las visiones del mundo de un inseguro yo" (Gimeno, p. 87).

Este cambio de relaciones, experimentado por los estudiantes en su paso a la secundaria, puede ser positivo o bien traumático; dependiente de las habilidades que han desarrollado desde la escuela primaria.

El estudio de Graham y Hill (2003) indica que los estudiantes del sétimo grado destacan el hacer nuevos amigos como uno de los tres elementos más importantes al ingresar a este nivel. Razón por la cual, la amistad y las relaciones interpersonales entre pares juega un papel fundamental en la transición y, por ende, exige una consideración por parte de docentes, familiares y autoridades gubernamentales.

Por otra parte y desde una perspectiva más traumática, Gimeno (1997) indica que al ingresar a la secundaria, el alumno, en la mayoría de las ocasiones, se ve forzado a romper lazos con sus compañeros de primaria, pues, generalmente, está de por medio un cambio de institución. Esta 
circunstancia causa, en el estudiante, una sensación de ruptura con su antigua vida y con sus anteriores esquemas para establecer relaciones interpersonales.

Para Craig, citado por Bolaños y Castro (2001), la amistad, después de los 11 años de vida, es vista por las personas como una relación estable y duradera, donde la persona logra asumir la posición del otro, es decir, se consolida el desarrollo pleno de la empatía. Aunado a lo anterior, desde la perspectiva de Gimeno (1997), las amistades adquieren un valor trascendental de apoyo a las inseguridades que definen esta etapa de desarrollo; "el grupo de semejantes forma un ámbito de referencia capital para el establecimiento de la identidad todavía titubeante" (p. 87). Por ende la amistad constituye un refugio a las necesidades de los adolescentes en un momento en que las relaciones de amistad son la base de la seguridad.

Desde esta perspectiva, Gimeno (1997) afirma que la transición a la secundaria supone la pérdida o dificultad para mantener amistades antiguas y la necesidad de entablar otras nuevas; sin embargo, el autor indica que todos esos cambios o rupturas experimentados por los adolescentes se dan al margen de la intervención de las instituciones educativas, es decir, no se incluye en el currículo formal un tiempo para construir nuevas amistades. En el mejor de los casos, el primer día se realizan unas actividades de inducción o "rompe hielo" y, por lo general, no se retoma este aspecto socioafectivo.

A manera de síntesis, se afirma que los lazos de amistad son de suma importancia para los adolescentes, durante el proceso de transición de la primaria a la secundaria; por tanto, la separación de sus compañeros y amigos de la escuela primaria les afecta para el establecimiento de nuevos lazos de amistad, primordialmente, porque no son preparados ni para la ruptura afectiva anterior, ni para las exigencias de las nuevas relaciones que están por iniciar.

Por lo anterior, se considera vital contemplar la realización de actividades sociales que proporcionen la oportunidad a los estudiantes de conseguir y desarrollar relaciones positivas con sus pares; o bien, con los estudiantes mayores o de otros niveles de la institución. Esto, debido a la trascendencia de las relaciones afectivas en este momento de la vida de los estudiantes y a la interrupción que causa la transición a las redes de amistad, factor de éxito de los estudiantes en la escuela secundaria.

\section{Relaciones de los estudiantes con los docentes en el proceso de transición}

Kirkpatrick (1997) indica que "Demasiado a menudo los profesores y el académico se olvidan de que el aprender tiene un componente afectivo importante y los estudiantes tienen respuestas afectivas a los profesores, a los pares, al contexto, a todos los aspectos académicos de la vida de la escuela. Estas respuestas afectivas junto con sus expectativas, motivación y funcionamiento académico influyen en sus creencias" (p. 1).

En el proceso de transición a la secundaria, los estudiantes experimentan una serie de cambios que incluyen, entre otros aspectos, pasar de relaciones unipersonales con un solo docente, a relaciones más complejas y menos personalizadas con doce o bien catorce profesores; quienes, a su vez, interactúan hasta con cuatrocientos estudiantes en una sola semana. Por esta razón, llamar a un estudiante por su nombre, o bien, darle seguimiento en su rendimiento académico o personal parece convertirse en una tarea compleja para el docente y para el estudiante, quien debe aprender a independizarse. La independencia, desde la perspectiva de Gimeno (1997), tiene que ver con el hecho de que los estudiantes pasan de un sistema maternalista a un ambiente que les exige mayor independencia y control, situaciones que pueden, inclusive, provocar crisis socioafectivas. 
Por tanto, las relaciones interpersonales que establecen los estudiantes adolescentes con sus profesores se modifican y esto los obliga a independizarse de manera rápida, brusca y creciente. Además, de verse obligados a ser cada día más independientes en sus tareas escolares, la bibliografía señala que los alumnos, en su paso a la secundaria, consideran que las posibilidades que les ofrecen sus profesores para expresarse libremente y tomar decisiones se restringen.

Con respecto a la toma de decisiones, Midgley y Feldlaufer (1987) realizan un estudio con estudiantes en el sétimo de secundaria, donde los estudiantes opinaron que se les debió permitir tomar más decisiones de las permitidas e indicaron que, comparado con su primer año de secundaria, durante su estadía en la primaria, pudieron tomar mayor cantidad de decisiones.

Para lograr que los estudiantes participen de manera más activa en la toma de decisiones, al ingresar a la secundaria, Midgley y Urdan (1992) señalan que tanto los administrativos como docentes pueden dar la oportunidad al alumnado de tomar decisiones acerca de sus propios horarios y regulaciones dentro del salón de clases. Este cambio en al ambiente de aprendizaje influye en el cambio motivacional sufrido por los estudiantes durante su proceso de transición, de ahí que las características de dicho ambiente juegan un papel muy importante (Midgley, 1993).

Sin embargo, esta suposición ha sido cuestionada por investigaciones que demuestran que la naturaleza del cambio motivacional, a la entrada de la escuela secundaria, depende de las características del ambiente de aprendizaje en el cual los estudiantes se encuentran (Midgley, 1993).

En este proceso de establecer relaciones interpersonales, Anderman y Midgley (citados por McGee, Ward, Gibbons y Harlow, 2003) indican que es indispensable que el docente asuma un rol que le permita entender lo que creen los estudiantes, saber escucharlos, ir más allá de éxitos y faltas, comunicarse asertivamente y respetarlos; esto, sin dejar que sus preconcepciones y juicios se inmiscuyan y afecten el desarrollo de su clase.

\section{Autoestima}

Para Papalia, Wendkos y Duskin (2001), la autoestima o autoconcepto se desarrolla desde la niñez. El crecimiento cognoscitivo le permite al infante tener una idea más realista y compleja de sí mismo y de las capacidades que posee. Además, se suma un mayor entendimiento y control de las emociones que experimentan los infantes conforme crecen y llegan a la adolescencia. Las mismas autoras indican que el autoconcepto se define como "Sentido de sí mismo; imagen mental descriptiva y evaluativa de las capacidades y rasgos de sí mismo" (p. 147).

Para González-Pienda, Núñez, Glez-Pumariega y García (1997), el autoconcepto es una variable fundamental en el desarrollo de la personalidad; por otra parte, estos autores insisten en que, en términos de estructura, la autoestima es más compleja en la adolescencia, por cuestiones propias de la edad. Es decir, que durante este periodo los jóvenes sufren una serie de cambios físicos, emocionales y sociales, los cuales afectan directamente su autoestima, de ahí que resulta necesario generar estrategias que favorezcan su concepto de sí mismos en este grupo etario. Por último, los autores indican que la capacidad para analizarse y conocerse también ha sido destacada actualmente por Gardner (1995). Este estudioso, dentro de su teoría de las inteligencias múltiples incluye la inteligencia intrapersonal como una de las siete inteligencias humanas; esta se refiere al conocimiento de uno mismo y todos los procesos relacionados tales como autoconfianza y automotivación.

Unido a las ideas anteriores sobre autoestima, Gimeno (1997) indica que la opinión de las otras personas significativas se convierte en un pilar fundamental en la construcción de la autoestima, 
debido a que "El proceso de la configuración de la autoimagen adquiere especial significado en la etapa de consolidación de la identidad del adolescente" (p. 83).

Desde otra perspectiva, Vera y Ribón (s. f.) consideran que "La autoestima medida mediante la valoración que los jóvenes perciben en el entorno acerca de su mayor o menor inteligencia, se asocia con el logro escolar: a medida que subimos en la clasificación fracaso, duda y éxito, aumenta el porcentaje de los que creen que el entorno los valora" (p. 3).

Por otra parte, Psaltis (2002), en una investigación longitudinal realizada con 157 estudiantes de primer ingreso a la secundaria, en una de sus conclusiones plantea que la principal preocupación de dichos estudiantes es realzar el concepto de sí mismos, por encima de las cuestiones de índole académica.

Para Cotterell (1986), la transición de la primaria a la secundaria genera, en los adolescentes, momentos difíciles, pues se encuentran en una etapa de su vida muy sensible, en la que se tornan agitados, nerviosos y bulliciosos, además, en la parte emocional experimentan un bajo concepto de sí mismos.

En un estudio realizado por Simmons, Rosenberg y Rosenberg (citados por Mullins e Irvin, 2000) con dos mil estudiantes que cursaban el sexto grado y el sétimo de secundaria, determinaron que la declinación más baja en la autoestima la sufren los estudiantes de sexto grado. Además, los mismos autores señalan que los alumnos del sétimo nivel manifestaron creencias relacionadas con el hecho de que los padres, profesores y sus pares tenían una visión menos favorable de ellos.

Desde otra perspectiva, Harter, Rumbaugh y Kowalski (1992) reportan, en su investigación, que los estudiantes cuyo rendimiento académico fue muy bajo en el sexto grado enfrentaron el proceso de transición con mayores niveles de estrés, lo cual les provocó menor motivación y una declinación más aguda en su autoestima, situación que ocurrió en menor proporción con los estudiantes cuyo rendimiento académico era superior.

Según Gimeno (1997), las consecuencias directas en la autoestima de los estudiantes, al cursar el sétimo año de secundaria, se relacionan con el cambio de ambiente, lo cual les exige un nuevo posicionamiento, es decir, pasan de un grupo en el que son quizás los más responsables y populares, a un grupo desconocido de amigos y profesores, en el cual hay alumnos más seleccionados y son evaluados más severamente. Este mismo autor manifiesta que, en los estudios que ha realizado, el $45 \%$ de los estudiantes del nivel inicial de la secundaria sufre declinaciones en autoconcepto académico, lo cual se relaciona con los problemas en el rendimiento académico, por ausencia de motivación hacia el estudio.

Unido al tema del autoconcepto, Gimeno (1997) indica que la seguridad, es decir, el convencimiento del estudiante de realizar el paso a la secundaria y mantenerse es fundamental para el logro del mismo. Por otra parte, dice el autor que la esperanza de pasar y seguir en la secundaria para los alumnos se relaciona directamente con el nivel de escolaridad de los padres, ya que cuanto más elevado sea, aumentan las posibilidades de ellos para superar esta etapa.

En términos generales, los estudios evidencian que la autoestima, además de ser una variable que interviene en el desarrollo de la personalidad, también, figura como elemento importante en la motivación y se convierte, en la transición de la primaria a la secundaria, en un factor muy importante, porque los adolescentes inician una etapa de desarrollo evolutivo, muy marcada por necesidades de autonomía, consolidación de la personalidad, diferenciaciones importantes de las características de sexo (hombre-mujer) y cambios importantes en el marco y forma de relacionarse socialmente con sus pares. De ahí que es imperante establecer mayor coordinación entre los programas escolares y colegiales, y considerar en ellos las necesidades propias de la población hacia la cual se dirigen. 


\section{Los valores y la adolescencia}

La primera pregunta que salta al respecto es: ¿de dónde toman los adolescentes los valores? Evidentemente, los valores se adquieren en la familia, la sociedad y la escuela.

Los estudiosos de este tema prefieren abordar, en forma separada, la etapa de la adolescencia, debido al impacto y gran cantidad de cambios que se dan tanto en los adultos como en los mismos adolescentes. En esta etapa, los hijos se plantean el por qué, el para qué, el sentido de la vida, qué quieren hacer, cómo quieren vivir. A través de estas preguntas, el adolescente también "mueve" a los padres, y los lleva a replantearse sus propias opciones al respecto. Pueden darse conflictos de valores y enfrentamientos, con la diferencia de que el adolescente tiene tiempo para solucionarlos, mientras que los padres no. Esta etapa puede resolverse mediante el control por parte de los padres o, por el contrario, por una indiscriminación entre ellos y sus hijos, de manera que funcionan como amigos.

Por lo general, los valores que poseen las personas y, principalmente los adolescentes, los distinguen ante otras personas. Esta presunción de valores se hace con base en la observación. Desde luego la apariencia externa es importante, pero sólo como eso, como lo que hace parecer, lo que demuestra el interior del muchacho es lo que realmente tiene gran valor (Campos, 2001).

Algunos rasgos distintivos de los valores que posee un estudiante van desde el aprecio que tiene de sí mismo, cuánto respeta a los demás, hasta la falta de aceptación de los otros -que muchas veces se refleja en su arreglo personal- y conductas que él conoce muy bien como no aceptadas ni por la familia ni por la escuela (Campos, 2001).

La adolescencia es un período del desarrollo humano en el cual se forman los valores que no se adquirieron en la niñez, se corrigen los que se adquirieron, o bien se forman de manera definitiva. Por esto, son de enorme importancia las palabras (como expresiones de ideas y conceptos) y actitudes de los agentes de la comunidad educativa, en especial las de los docentes (Campos, 2001).

De la Fuente, Peralta y Sánchez (2006) indican que durante la convivencia escolar los estudiantes pueden mostrar conductas que oscilan desde una adecuada hasta una inadecuada adaptación, lo cual influye en el logro de metas académicas que persiguen los mismos estudiantes. Además, estos autores hacen referencia a estudios que coinciden en que la desmotivación y el desinterés académico influyen en la autoestima, competencia social e identificación con el grupo de iguales de los estudiantes. Adicionalmente aseguran que las conductas agresivas en el contexto escolar pueden generar problemas a los alumnos en su adolescencia, de ahí que es indispensable trabajar los valores en relación directa con la convivencia escolar.

Los docentes deben ser cuidadosos para no dejarse llevar por las conductas observables que conllevan un juicio, aunque estos juicios se basan en normas previamente establecidas y aceptadas por la comunidad. Los valores como práctica, además de regular la conducta, la guían y la ordenan de acuerdo con una escala de valores tanto personal y como social (Campos, 2001).

Los valores en el trabajo cotidiano de clase pueden ser abordados positivamente, si se asegura que el docente establezca relaciones interpersonales con sus estudiantes, basadas en la comunicación asertiva, el respeto y la tolerancia. Por otra parte, es indispensable que se implementen estrategias metodológicas que permitan una aprehensión de los valores a los estudiantes, con el objetivo de que su proyecto de vida esté permeado de valores universalmente construidos.

Las relaciones interpersonales, autoestima y valores que construye el estudiante durante su paso de la primaria a la secundaria, tal y como lo indica la bibliografía, son determinantes en este proceso de cambio, porque influyen, de alguna manera, en su percepción acerca de sus posibilidades de éxito o fracaso durante su estadía en la secundaria. 
En los siguientes apartados se caracteriza a los participantes de este estudio y se presentan datos que confirman la importancia de establecer relaciones interpersonales positivas para los estudiantes, durante su paso a la secundaria.

\section{Aspectos metodológicos}

\section{Tipo de investigación}

La presente investigación es uno de los primeros esfuerzos, a nivel costarricense, que tiene como uno de sus objetivos recabar la opinión de los alumnos y las alumnas de $6^{\circ}, 7^{\circ}$ y $8^{\circ}$ años respecto a lo que ellos y ellas consideran como los factores claves para lograr una transición exitosa entre primaria y secundaria, por tanto, se considera un estudio de carácter exploratorio, ya que sus resultados permitirán el logro de un acercamiento o aproximación al tema y servirá, además, de punto de partida para otros trabajos de investigación vinculados a esta temática.

Por otra parte, tiene un carácter descriptivo, al pretender identificar, a partir de opiniones, criterios y observaciones, los elementos o rasgos claves que caracterizan la situación de los colegios considerados más exitosos en cuanto a permanencia y avance en el sistema educativo.

Asimismo, utiliza, en forma complementaria, técnicas para la recolección de los datos tanto cuantitativas, como cualitativas; por otra parte, este estudio no es representativo de la situación nacional de todos los colegios; sin embargo, por medio del mismo se brinda un panorama de los factores asociados al éxito en los 10 colegios seleccionados.

\section{Participantes}

En la investigación sobre transición de la primaria a la secundaria, se seleccionaron 10 instituciones de educación secundaria, las cuales permiten trabajar con 1671 alumnos de sétimo y octavo años, además de 64 docentes. Detalles de la selección se indican en las siguientes líneas.

Para seleccionar las 10 instituciones de educación secundaria que participan en la investigación, se determinan los siguientes criterios:

- Estadísticas de alto rendimiento académico y baja deserción.

- Ubicación geográfica (pertenecientes a diferentes zonas del país, valle central y zonas alejadas).

La combinación de los criterios "alto rendimiento académico" y "baja deserción escolar" es, desde la perspectiva de los investigadores, una fórmula que permite identificar el éxito en los procesos de enseñanza y aprendizaje que experimentan los estudiantes, ya que las instituciones no solo organizan sus esfuerzos para lograr alto rendimiento académico de sus estudiantes, sino también disponen de estrategias para lograr su permanencia a lo largo del ciclo lectivo.

Otro de los criterios para la selección de las instituciones educativas participantes se refiere a su ubicación geográfica, es decir, se consideró como criterio importante que pertenecieran a diferentes zonas geográficas del país, con la intención de comprender desde contextos diversos el tema en estudio. Los centros educativos se ubicaron en zonas tales como Liberia, Cañas, 
Desamparados, Pérez Zeledón, Guápiles, Santa Bárbara (Heredia), Cartago (cantón central), Grecia, Pavas y Turrialba. Por otra parte, estos centros educativos son, en su mayoría, públicos y, solamente en un caso, de tipo experimental. Asimismo, todos tienen rendimientos superiores a la media nacional.

De cada uno de los centros educativos seleccionados se trabajó con el total de estudiantes de sétimo y octavo años, lo anterior implicó 853 adolescentes de sétimo año y 818 estudiantes de octavo, para un total de 1671 alumnos. También se contó con la participación de 33 docentes de sétimo y 31 de octavo años.

\section{Instrumentos}

Los instrumentos utilizados para el logro del objetivo propuesto y la estrategia de aplicación se indican en la siguiente tabla:

Tabla 1

Descripción de los instrumentos y estrategias de aplicación

\begin{tabular}{|c|c|c|c|}
\hline $\begin{array}{l}\text { Nombre del } \\
\text { instrumento }\end{array}$ & $\begin{array}{l}\text { Participantes a } \\
\text { quienes se les aplica }\end{array}$ & $\begin{array}{l}\text { Aspectos que considera el } \\
\text { instrumento }\end{array}$ & Estrategia de aplicación \\
\hline $\begin{array}{l}\text { Cuestionario para } \\
\text { estudiantes de } 7 \text { y } 8 \\
\text { años }\end{array}$ & $\begin{array}{l}1671 \text { estudiantes, } \\
\text { distribuidos en } 853 \\
\text { adolescentes de } \\
\text { sétimo año y } 818 \\
\text { estudiantes de octavo } \\
\text { año }\end{array}$ & $\begin{array}{l}\text { - Preferencias respecto a } \\
\text { materias y profesores. } \\
\text { - Percepción del proceso } \\
\text { de transición (fortalezas y } \\
\text { debilidades). } \\
\text { - Características personales. } \\
\text { - Autoestima. } \\
\text { - Aspectos afectivos. } \\
\text { - Relaciones interpersonales. }\end{array}$ & $\begin{array}{l}\text { Autoadministrado } \\
\text { (Hernández, Fernández } \\
\text { y Baptista, 2003): Los } \\
\text { instrumentos fueron } \\
\text { aplicados por los } \\
\text { investigadores directamente } \\
\text { a los estudiantes, quienes } \\
\text { se encargan de responder } \\
\text { directamente a las } \\
\text { preguntas. }\end{array}$ \\
\hline $\begin{array}{l}\text { Cuestionario para } \\
\text { docentes de sétimo año }\end{array}$ & $\begin{array}{l}33 \text { docentes que } \\
\text { imparten lecciones en } \\
\text { sétimo nivel }\end{array}$ & $\begin{array}{l}\text { - Factores personales y } \\
\text { académicos que favorecen } \\
\text { la transición. } \\
\text { - Relaciones interpersonales } \\
\text { con estudiantes. } \\
\text { - Papel de los padres de } \\
\text { familia en el proceso de } \\
\text { transición. }\end{array}$ & $\begin{array}{l}\text { Autoadministrado } \\
\text { (Hernández, Fernández } \\
\text { y Baptista, 2003): el } \\
\text { cuestionario fue contestado } \\
\text { por docentes directamente }\end{array}$ \\
\hline $\begin{array}{l}\text { Cuestionario para } \\
\text { docentes de sétimo año }\end{array}$ & $\begin{array}{l}31 \text { profesores de } \\
\text { octavo año }\end{array}$ & $\begin{array}{l}\text { - Factores personales y } \\
\text { académicos que favorecen } \\
\text { la transición. } \\
\text { - Relaciones interpersonales } \\
\text { con estudiantes . } \\
\text { - Papel de los padres de } \\
\text { familia en el proceso de } \\
\text { transición. }\end{array}$ & $\begin{array}{l}\text { Autoadministrado } \\
\text { (Hernández, Fernández } \\
\text { y Baptista, 2003): el } \\
\text { cuestionario fue contestado } \\
\text { por docente directamente }\end{array}$ \\
\hline
\end{tabular}




\section{Validación de los instrumentos}

El cuestionario para estudiantes de 7 y 8 año fue validado por criterio de jueces. Además, se realiza un pretest (Cea, 1999) con 80 de alumnos externos a la muestra, que asistían al sexto grado y primer año de secundaria, en instituciones públicas, pertenecientes al circuito 02 de la provincia de Heredia. Posteriormente se analizan los resultados obtenidos y se descartan aquellos ítemes que generan respuestas ambiguas.

Para validar el cuestionario de docentes, este se suministró a 15 maestros en servicio, quienes funcionaron como jueces del mismo. Posteriormente se analizan los instrumentos desde una matriz que permite identificar los ítemes que requieren aclarase o bien eliminarse. Luego, a manera de pretest, se aplican nuevamente a un grupo de docentes (Cea, 1999).

Para el análisis de los resultados, se procede a combinar datos de frecuencias y porcentajes con categorías emergentes para las preguntas de carácter abierto, razón por la cual, en algunos de los cuadros se registra como total el número de participantes y en otros casos el número de respuestas, las cuales oscilaron entre 2 ó 3 como máximo.

Por otra parte, algunos cuadros identifican información de estudiantes de octavo año. Esto se justifica en la premisa del grupo investigador, respecto a que la transición es un proceso y, por tanto, en este grupo de participantes pueden identificarse algunos rasgos que permiten comprenderlo desde una perspectiva más amplia e integral.

\section{Presentación y discusión de los resultados}

Las relaciones interpersonales que establecen los estudiantes con sus iguales y con sus profesores es un factor de gran importancia durante el proceso de transición, ya que enfrentar un ambiente con más de un profesor y nuevos compañeros y amigos representa un temor para los estudiantes de sexto grado de primaria y un reto para los de sétimo, según se establece en diferentes investigaciones (Graham y Hill, 2003). Para indagar sobre cómo se enfrenta este reto, se consultó a los participantes acerca de los aspectos afectivos que intervienen en las relaciones que ellos establecen. Al respecto, los datos indican que al ingresar a la secundaria e inclusive en el octavo año (II nivel de secundaria), los estudiantes califican las relaciones con sus padres, compañeros y docentes como positivas, especialmente las que establecen con sus padres. Esta afirmación se demuestra en la tabla 2.

Tabla 2

Tabla comparativa sobre la valoración de los estudiantes de $7^{\circ}$ y $8^{\circ}$ nivel de secundaria acerca de las relaciones interpersonales que establecen

\begin{tabular}{|c|c|c|c|c|c|c|}
\hline \multirow[b]{2}{*}{ Nivel } & \multicolumn{2}{|c|}{ Nunca/Casi nunca } & \multicolumn{2}{|c|}{ A veces } & \multicolumn{2}{|c|}{ Siempre/Casi siempre } \\
\hline & $7^{\circ}$ & $8^{\circ}$ & $7^{\circ}$ & $8^{\circ}$ & $7^{\circ}$ & $8^{\circ}$ \\
\hline $\begin{array}{l}\text { Tengo buenas relaciones con los } \\
\text { compañeros }\end{array}$ & 3,0 & 3,0 & 21,0 & 18,5 & 76,0 & 78,5 \\
\hline Tengo buenas relaciones con los profesores & 4,5 & 4,1 & 25,2 & 28,2 & 70,3 & 67,8 \\
\hline Tengo buenas relaciones con los familiares & 3,3 & 3,8 & 9,7 & 10,4 & 87,0 & 85,8 \\
\hline
\end{tabular}

Nota. Cuestionario para estudiantes de 7 y 8 año. 
Si bien es cierto, los datos de la tabla 2 indican que los estudiantes tienen buenas relaciones con sus padres y profesores mientras cursan el primer y segundo año de secundaria, los de la tabla 3 evidencian que, en un momento de crisis o bien al enfrentar una situación problema, la figura del amigo o amiga resulta de gran importancia para el estudiante. Estos datos confirman lo expresado por Gimeno (1997), quien indica que "En el grupo de iguales el adolescente encuentra lealtad, un clima en el que expresar con confianza conflictos y las visiones del mundo de un inseguro yo" (p. 87). Además, "Para unos estudiantes la transición supone relaciones más maduras con sus profesores, como si fuesen "de adulto a adulto", hasta con más camaradería" (Gimeno, p. 168). Este cambio en la relación puede causar que los estudiantes prefieran a sus iguales al establecer relaciones interpersonales, incluso por sobre los adultos, durante el proceso de transición a la secundaria.

Tabla 3

Tabla comparativa acerca de la persona con la cual prefieren compartir los problemas los estudiantes, según nivel que cursan

\begin{tabular}{lcc}
\hline Nivel/Persona & $7^{\circ}$ Nivel & $8^{\circ}$ Nivel \\
\hline Amigo y o amiga & $59,3 \%$ & $62,6 \%$ \\
Familiar & $34,0 \%$ & $35,8 \%$ \\
Profesor & $1,8 \%$ & $1,6 \%$ \\
No responde & $4,9 \%$ & $0,0 \%$ \\
Total & $100 \%$ & $100 \%$ \\
\hline
\end{tabular}

Nota. Cuestionario para estudiantes de 7 y 8 año.

El amigo o amiga, como persona de confianza del adolescente en el proceso de transición, es también reconocido por los docentes consultados, quienes, en su gran mayoría, concuerdan en que el compañero o bien el amigo es la persona, por excelencia, con quien establece relaciones el estudiante al ingresar al sétimo de secundaria. Lo anterior reitera lo mencionado en el referente teórico, sobre la importancia y necesidad de abrir espacios en el currículo formal para el acercamiento y la conformación de lazos de amistad entre los estudiantes, dado que esto constituye un factor de éxito y permanencia de los estudiantes en la secundaria, es decir, la amistad es un refugio a las necesidades de los adolescentes en un momento en que las relaciones de amistad son la base de la seguridad.

Tabla 4

Tabla Calificación de las relaciones de los estudiantes con otros miembros de la comunidad educativa según los docentes y las docentes

\begin{tabular}{|c|c|c|c|c|c|c|c|c|}
\hline \multirow[t]{2}{*}{ Categoría } & \multicolumn{2}{|c|}{$\begin{array}{c}\text { Muy } \\
\text { importantes }\end{array}$} & \multicolumn{2}{|c|}{$\begin{array}{l}\text { Bastante } \\
\text { importantes }\end{array}$} & \multicolumn{2}{|c|}{$\begin{array}{c}\text { Algo } \\
\text { importantes }\end{array}$} & \multicolumn{2}{|c|}{$\begin{array}{c}\text { Nada } \\
\text { importantes }\end{array}$} \\
\hline & $7^{\circ}$ & $8^{\circ}$ & $7^{\circ}$ & $8^{\circ}$ & $7^{\circ}$ & $8^{\circ}$ & $7^{\circ}$ & $8^{\circ}$ \\
\hline Los compañeros y compañeras & 22 & 25 & 9 & 4 & 2 & 2 & 0 & 0 \\
\hline Los profesores y profesoras & 21 & 15 & 5 & 9 & 7 & 7 & 0 & 0 \\
\hline El director o directora & 17 & 14 & 6 & 4 & 7 & 8 & 3 & 5 \\
\hline El profesor (a) guía & 24 & 20 & 7 & 5 & 2 & 6 & 0 & 0 \\
\hline
\end{tabular}

Nota. Cuestionario para docentes de sétimo año, Cuestionario para docentes de octavo año. 
Las relaciones interpersonales de los estudiantes con sus compañeros, profesores y otros funcionarios del centro educativo, constituyen un factor relevante en el éxito y permanencia de los estudiantes en el centro educativo, tal y como se establece en la bibliografía (Gimeno, 1997; Kirkpatrick, 1997). Por otra parte, los datos de la tabla anterior evidencian que desde la perspectiva de los docentes, ellos se convierten en una figura importante para sus estudiantes al momento de establecer relaciones interpersonales.

A los profesores también se les consultó acerca del componente del desarrollo humano al que dan mayor importancia y el 59\% asegura que lo sociofectivo es fundamental y algunas de las razones que anotaron como justificación a esta consideración fueron las siguientes: "Es básica la relación docente estudiante para motivar el conocimiento de debilidades y fortalezas, hecho que quizá ayuda mucho más al éxito escolar del estudiante", "Muchas veces el interesarse por ellos les asienta muy bien y se sienten importantes". Estos datos complementan la visión de los profesores y evidencia, una vez más, que las relaciones interpersonales y lo afectivo resultan vitales para adaptarse a la secundaria.

Cualificar a un profesor como "bueno" de acuerdo con los datos de la investigación también se relaciona con el factor afectivo y las relaciones interpersonales que establece el estudiante, según se observa en la siguiente tabla.

Tabla 5

Tabla Razones por las que los escogieron como los mejores profesores, según nivel que cursa desde la visión de los estudiantes

\begin{tabular}{|c|c|c|c|c|}
\hline \multirow[t]{2}{*}{ Nivel/categoría } & \multicolumn{2}{|c|}{$7^{\circ}$ Nivel } & \multicolumn{2}{|c|}{$8^{\circ}$ Nivel } \\
\hline & Frecuencia & Porcentaje & Frecuencia & Porcentaje \\
\hline Relación personal & 821 & $34,09 \%$ & 654 & $28,71 \%$ \\
\hline Metodología & 712 & $29,57 \%$ & 781 & $34,28 \%$ \\
\hline Explicaciones & 357 & $14,83 \%$ & 427 & $18,74 \%$ \\
\hline Interés personal por el estudiante & 166 & $6,89 \%$ & 150 & $6,58 \%$ \\
\hline Gusto por la materia & 213 & $8,85 \%$ & 202 & $8,88 \%$ \\
\hline Importancia de la materia & 136 & $5,65 \%$ & 57 & $2,50 \%$ \\
\hline Otros & 3 & $0,12 \%$ & 7 & $0,31 \%$ \\
\hline Total de respuestas y porcentaje & 2408 & $100 \%$ & 2278 & $100 \%$ \\
\hline
\end{tabular}

Nota. Cuestionario para estudiantes de 7 y 8 año.

Entre las razones que aportan los estudiantes de ambos niveles para categorizar a sus mejores profesores como tales, se identifica una percepción altamente orientada a resaltar el aspecto de relaciones interpersonales, principalmente los alumnos que cursan el sétimo nivel, ya que los de octavo se inclinan por el aspecto metodológico, aunque colocan en segundo lugar la relación personal con su profesor. Ambos criterios (la metodología y la relación interpersonal) demuestran la importancia que tiene el papel de la motivación extrínseca, tanto en las estrategias didácticas como en las manifestaciones afectivas con los estudiantes, en los procesos de enseñanza-aprendizaje para potenciar alumnos exitosos.

Por otra parte, de la tabla 5 se desprende que el establecimiento de relaciones interpersonales adecuadas es muy importante para los estudiantes de sétimo nivel, quienes vivencian el proceso de transición, por tanto, un académico de secundaria debe cumplir con este requisito como parte de su perfil al momento de enfrentar con sus alumnos el tránsito de la primaria a la secundaria. Asimismo, 
los alumnos que cursan el octavo nivel y que culminan su transición a la secundaria, aseguran que las relaciones con el profesor son un criterio muy importante para una valoración positiva de su labor como docente. Esto indica que el ingreso a la secundaria es un espacio para que los alumnos desarrollen otras maneras de relacionarse y el profesor, por tanto, se convierte en una figura de gran valor.

En términos generales, se concluye que las relaciones interpersonales se convierten en un factor importante para el estudiante al ingresar a la secundaria, asimismo, los padres, amigos y docentes se consideran figuras determinantes al momento de establecer dichas relaciones.

Otros aspectos que se desarrollaron en esta investigación y que se vinculan directamente con las relaciones interpersonales, se refieren a los temas del autoconcepto y autoestima, así como también a los valores de los estudiantes, ya que estos condicionan la manera en que ellos se relacionan entre sí y con otras personas.

El autoconcepto es una variable fundamental en el desarrollo de la personalidad, por otra parte, durante la adolescencia los jóvenes sufren una serie de cambios físicos, emocionales y sociales, los cuales afectan directamente su autoestima, de ahí que es necesario generar estrategias que favorezcan el concepto de sí mismos.

Unido a la idea anterior, Gimeno (1997) indica que la opinión de los otros significativos constituye un pilar fundamental en la construcción de la autoestima, lo anterior debido a que "El proceso de la configuración de la autoimagen adquiere especial significado en la etapa de consolidación de la identidad del adolescente" (p. 83).

La autoimagen de los estudiantes de sétimo año de secundaria, de acuerdo con los datos de la investigación realizada que se exponen en la tabla 5, es positiva y, por tanto, les genera a ellos alto grado de aceptación de sí mismos, lo cual repercute en la seguridad que manifiesta un porcentaje importante de los alumnos de no ser rechazados e, inclusive, de convertirse "a veces" o "casi siempre" en el confidente de un amigo para compartir sus problemas. Por tanto, la percepción del otro es de gran importancia para el adolescente y su desenvolvimiento social, así como un factor de gran relevancia para quienes experimentan el proceso de transición de la primaria a la secundaria.

Tabla 6

Tabla Percepción que tienen los estudiantes de sétimo año de secundaria acerca de sí mismos, durante el proceso de transición de la primaria a la secundaria

\begin{tabular}{lcccc}
\hline Categorías & Nunca/casi nunca & A veces & Casi siempre/siempre & Total \\
\hline Me acepto como soy & $2,5 \%$ & $8,6 \%$ & $88,9 \%$ & $100 \%$ \\
Merezco ser amado & $2,7 \%$ & $10,7 \%$ & $86,6 \%$ & $100 \%$ \\
Tengo miedo al rechazo de mis amigos & $59,6 \%$ & $24,0 \%$ & $16,40 \%$ & $100 \%$ \\
Me buscan cuando tienen problemas & $24,1 \%$ & $51,8 \%$ & $24,1 \%$ & $100 \%$ \\
\hline
\end{tabular}

Nota. Cuestionario para estudiantes de 7 año.

Para tener una compresión más amplia de la percepción de sí mismos que tienen los estudiantes, se les consultó acerca de sus aspiraciones futuras y los valores que consideran más importantes para el desarrollo de su vida, especialmente al enfrentar el paso de la primaria a la secundaria.

Los datos de la investigación presentados en la tabla 6 confirman que los alumnos encuestados tienen varias aspiraciones, dentro de las cuales se destacan ser profesional, laborar y terminar sus estudios. Cabe destacar que el 8,05\% de los encuestados indica que tener una familia es importante como proyecto de vida. 
Si bien es cierto, la tabla permite conocer las aspiraciones de los estudiantes, también asegura al lector que este grupo de jóvenes piensa en su vida futura de manera concreta, es decir, tiene metas y estas se relacionan con las aspiraciones mismas que la sociedad podría esperar de un grupo de jóvenes.

La literatura relacionada con la inteligencia emocional indica que el concepto del otro es muy importante en la construcción del autoconcepto, pero también lo que la persona piensa sobre sí misma.

Tabla 7

Tabla Aspiraciones futuras que tienen los estudiantes de sétimo nivel de secundaria durante el proceso de transición

\begin{tabular}{lcc}
\hline Categoría & Frecuencia & Porcentaje \\
\hline Ser profesional & 579 & 26,35 \\
Tener un trabajo & 436 & 19,85 \\
Ganar el bachillerato & 313 & 14,25 \\
Concluir los estudios & 241 & 11,00 \\
Tener una familia & 177 & 8,05 \\
Ser un deportista exitoso & 122 & 5,55 \\
Tener un proyecto de vida & 108 & 4,90 \\
Ser una buena persona & 88 & 4,00 \\
Ayudar a los padres & 58 & 2,63 \\
Ganar mucho dinero & 50 & 2,28 \\
Ser feliz & 25 & 1,14 \\
Total de respuestas & 2197 & $100 \%$ \\
\hline
\end{tabular}

Nota. Cuestionario para estudiantes de 7 año.

Además de evidenciar los proyectos y aspiraciones de los estudiantes al cursar el primer año de la secundaria, los cuales no solo reflejan la ubicación de ellos como adultos en el futuro cercano, esta investigación también logró acceder a los valores y sentimientos que este grupo de jóvenes conoce y la prioridad que tienen para ellos en su vida diaria.

Tabla 8

Tabla Principales valores y sentimientos que indican los estudiantes de sétimo y octavo para sus proyectos de vida

\begin{tabular}{lcccc}
\hline \multicolumn{1}{c}{ Nivel/Valores } & \multicolumn{2}{c}{ Sétimo Nivel } & \multicolumn{2}{c}{ Octavo Nivel } \\
\hline Respeto & Frecuencia & Porcentaje & Frecuencia & Porcentaje \\
Amor & 673 & $26,15 \%$ & 657 & $25,80 \%$ \\
Trabajo & 658 & $25,6 \%$ & 631 & $24,78 \%$ \\
Solidaridad & 316 & $12,31 \%$ & 277 & $10,87 \%$ \\
Convivencia & 307 & $11,93 \%$ & 352 & $13,82 \%$ \\
Tolerancia & 294 & $11,42 \%$ & 270 & $10,60 \%$ \\
Igualdad & 236 & $9,17 \%$ & 261 & $10,25 \%$ \\
Total de respuestas y porcentaje & 88 & $3,42 \%$ & 99 & $3,88 \%$ \\
\hline
\end{tabular}

Nota. Cuestionario para estudiantes de 7 y 8 año. 
Los datos del presente estudio revelan que los estudiantes, durante el periodo de transición, tanto al cursar el sétimo como el octavo nivel, mantienen el orden de prioridad que conceden a todos los valores y sentimientos mencionados en la tabla, asimismo, el respeto y el amor son considerados por los alumnos como fundamentales en sus vidas, lo cual evidencia no solo lo que están dispuestos a ofrecer en sus relaciones interpersonales en la secundaria durante la transición, sino también, lo que requieren para fortalecer su autoconcepto.

En un segundo bloque de datos los valores de trabajo, solidaridad, convivencia y tolerancia, se muestran como valores menos importantes, sin embargo, indispensables para el establecimiento de adecuadas relaciones interpersonales. La igualdad es identificada por los estudiantes como el valor menos importante en sus vidas.

En términos generales la investigación identificó que los jóvenes que viven un periodo de transición escolar tienen claridad de los valores que son importantes en sus vidas, asimismo, estos valores se relacionan con los proyectos o perspectivas futuras que construyen. Este conjunto de ideales y valores son el marco propicio para que docentes y autoridades institucionales reorienten los esfuerzos en materia de educación secundaria, especialmente porque, de acuerdo con los estudios sobre esta temática, la adolescencia es un período de consolidación de valores, de ahí la importancia de las palabras, expresiones y actitudes de los agentes de la comunidad educativa, en especial las de los docentes (Campos, 2001).

\section{Conclusiones y recomendaciones}

La investigación realizada permite concluir que, al ingresar a la secundaria, los alumnos mantienen adecuadas relaciones interpersonales con sus padres, docentes y compañeros. El amigo o amiga y el padre de familia son, para los estudiantes de sétimo nivel, figuras de apoyo muy importantes, especialmente cuando ellos enfrentan algún problema, es decir, se convierten en confidentes y ofrecen seguridad en un momento en que todo parece dar un giro y cambiar rápidamente, de ahí que velar por un clima de confianza y amistad parece ser una opción valiosa para los centros de educación secundaria, al momento de planificar la transición de los estudiantes de la primaria a la secundaria.

Las relaciones interpersonales son muy importantes en el desarrollo de todo ser humano y en el proceso de transición a la secundaria parecen cobrar un sentido especial para el estudiante, debido, primordialmente, a que se multiplican en comparación con las que establece el alumno en la primaria, de ahí que preparar al estudiante en la dimensión afectiva requiere de un plan de trabajo institucional y del reconocimiento de la parte afectiva y relacional en los procesos de enseñanza y aprendizaje.

Otro hallazgo importante del estudio radica en que el autoconcepto positivo y tener proyectos de vida definidos se combinaron en la vida de los jóvenes que participaron en esta investigación, para que el proceso de transición a la secundaria se considerara como exitoso para, al menos, el 50\% de los alumnos participantes. Este aporte de la investigación pone en evidencia que un plan de transición, al ser diseñado por miembros de la comunidad educativa, les exige conocer acerca de la dimensión afectiva y de los valores con que ingresan los muchachos al colegio, ya que ambos aspectos adecuadamente potenciados se convierten en factores de éxito en la transición de la primaria a la secundaria.

Como parte de la dimensión de las relaciones interpersonales, la investigación permite concluir que durante el proceso de transición a la secundaria, los estudiantes identifican una serie de valores 
tales como el respeto, el amor, la tolerancia y la convivencia como valores muy importantes, caracterizadores del tipo de relaciones que ellos establecen o desean establecer con las personas que integran el nuevo medio al que se enfrentan. Este hallazgo asegura que los jóvenes viven los valores y los categorizan de acuerdo con sus necesidades, razón por la cual enfatizar en estos es determinante para los profesores, quienes mantienen un contacto permanente y de alguna manera modelan dichos valores. Asimismo, en el proceso de transición, los valores colaboran para la definición del autoconcepto del estudiante, el cual tiende a declinar durante el proceso de transición.

En relación con la figura de los docentes, se determinó que los factores que diferencian a los profesores considerados como mejores de los peores se centra, para los alumnos de sétimo nivel, en las relaciones interpersonales. Los estudiantes que cursan el sétimo nivel valoran la parte humanista de sus docentes, relacionada con la necesidad de afectividad, por ser este el año de transición de un sistema maternal en primaria a otro pluridocente, multimetodológico y multidisciplinar.

En términos generales, los estudiantes durante la transición a la secundaria, se enfrentan a un ambiente de relaciones más amplio y complejo, que enfrentan, en primera instancia, recurriendo a la figura de sus padres y amigos cercanos. Asimismo, el carácter humano del docente no desaparece por el simple hecho de cambiar de institución o bien de la cantidad de profesores, es así como la necesidad de relacionarse con valores como el respeto y la tolerancia cobran gran importancia para un grupo de jóvenes, cuyo autoconcepto tiende a declinar y, por tanto, requiere de personas que le brinden una red de apoyos.

\section{Referencias bibliográficas}

Bolaños, A. y Castro, M. (2001). El concepto de amistad que tienen los niños y niñas de dos jardines infantiles en la provincia de Heredia. (Tesis de Licenciatura no publicada). Universidad Nacional, Heredia, Costa Rica.

Campos, O. (2001). En la educación básica ¿valores o virtudes? Revista Iberoamericana de Educación, 1-5. Recuperado de http://www.rieoei.org/deloslectores/Campos.PDF

Cea, M. (1999). Metodología cuantitativa. Estrategias y técnicas de investigación social. Madrid: Editorial Síntesis.

Cotterell, J. (1986). The Adjustment of Early Adolescent Youngsters to Secondary School: Some Australian Findings [Ajuste de los adolescentes jóvenes a la secundaria: resultados en Australia]. En M. B. Youngman (Ed.), Mid-Schooling Transfer: Problems and Proposals [Transición a la escuela media (sétimo y octavo año): problemas y propuestas] (66-86). Windsor: NFER-Nelson.

De la Fuente, J., Peralta, F. y Sánchez, M. (2006). Valores sociopersonales y problemas de convivencia en la educación secundaria. Revista Electrónica de Investigación Psicoeducativa 4(2), $171-200$.

Gardner, H. (1995). Reflections on Multiple Intelligences: Myths and Messages [Reflexiones sobre las inteligencias múltiples: mitos y mensajes]. Phi Delta Kappan, 77(3), 200-208. 
Gimeno, J. (1997). La transición a la educación secundaria. Madrid, España: Ediciones Morata.

González-Pienda, J., Núñez, J., Glez-Pumariega, S. y García, M. (1997). Autoconcepto, autoestima y aprendizaje escolar. Psicothema, 9(2), 271-289.

Graham, C. y Hill, M. (2003). Negotiating the transition to secondary school [La negociación en la transición a la secundaria]. University of Glasgow Edimburgo: Centro SCRE de la Universidad de Glasgow. Recuperado de http://www.pre-online.co.uk/feature pdfs/spotlight89.pdf

Harter, S., Rumbaugh, N. y Kowalski, P. (1992, diciembre). Individual Differences in the Effects of Educational Transitions on Young Adolescent's Perceptions of Competence and Motivational Orientation [Diferencias individuales de los efectos de las transiciones educativas en la percepción de jóvenes adolescentes de su competencia y actitud motivacional]. American Educational Research Journal, 29(4), 777-807. doi: 10.3102/00028312029004777

Hernández, R., Férnandez, C., Baptista, P. (2003). Metodología de la investigación. México, D.F. McGraw-Hill Interamericana.

Kirkpatrick, D. (1997). Making the Change: Students Experiences of the Transition to Primary School [Haciendo el cambio: experiencias de los estudiantes en la transición a la escuela primaria]. Recuperado de http://edoz.com.au/educationaustralia/archive/features/make.html

McGee, C., Ward, R., Gibbons, J. y Harlow, A. (2003). Transition to Secondary School: A Literature Review [Transición a la escuela secundaria: una revisión de la literatura]. Nueva Zelanda: Ministerio de Educación. Recuperado de http://www.educationcounts.govt.nz/publications/ $\underline{\text { schooling/5431 }}$

Midgley, C. y Feldlaufer, H. (1987). Students' and Teachers' Decision-Making Fit Before and After the Transition to Junior High School [Empate de las decisiones de estudiantes y profesores antes y despúes de la transición a la secundaria]. Journal of Early Adolescence, 7(2), 225241.

Midgley, C. y Urdan, T. C. (1992). The Transition to Middle Level Schools: Making it a Good Experience for all Students [La transición en el nivel de escuela media: una buena experiencia para todos los estudiantes]. Middle School Journal, 24(2), 5-14. EJ454359.

Midgley, C. (1993). Motivation and Middle Level Schools [Motivación y escuela media]. En P. R. Pintrich y M. L. Maehr (Eds.), Advances in motivation and achievement, Vol. 8: Motivation in the adolescent years [Avances en motivación y rendimiento, volume 8: motivación en la adolescencia] (219-276). Greenwich, CT: JAI Press.

Mullins, E. e Irvin, J. (2000, enero). Transition into Middle School [Transición a la escuela media (sétimo y octavo año]. Middle School Journal, 31(3), 57-60. Recuperado de www.nmsa.org/ portals/0/pdf/.../On.../transitioning.../transitioning ms 7.pdf 
Papalia, D., Wendkos, S., y Duskin, R. (2001). Psicología del desarrollo (8 ${ }^{\text {a }}$ ed.). Colombia: McGraw-Hill.

Psaltis, I. (2002). A longitudinal Survey into school transfer from primary to secondary education [Un estudio longuitudinal de la transición de la educación primaria a la secundaria]. Recuperado de http://schulen.eduhi.at/lipss/ manual2 11.htm

Vera, J. y Ribón, M. (s. f.). Éxito, fracaso y abandono escolar en la educación secundaria obligatoria. Análisis de la primera cohorte que culmina la E.S.O en el municipio de Puerto Real. Buenas tareas.com. Recuperado de http://www.buenastareas.com/ensayos/Éxito-Fracaso-yAbandono-Escolar-En/514630.html 